$1-1-1977$

\title{
Aquatic insects as indicators of stream environmental quality in northern West Virginia
}

Bonnie Wojcik

Linda Butler

Follow this and additional works at: https://researchrepository.wvu.edu/ wv_agricultural_and_forestry_experiment_station_bulletins

\section{Digital Commons Citation}

Wojcik, Bonnie and Butler, Linda, "Aquatic insects as indicators of stream environmental quality in northern West Virginia" (1977). West Virginia Agricultural and Forestry Experiment Station Bulletins. 653.

https://researchrepository.wvu.edu/wv_agricultural_and_forestry_experiment_station_bulletins/554 @ WVU. It has been accepted for inclusion in West Virginia Agricultural and Forestry Experiment Station Bulletins by an authorized administrator of The Research Repository @ WVU. For more information, please contact ian.harmon@mail.wvu.edu. 


\section{quatic Insects as Indicators of tream Environmental Quality Northern West Virginia}

lletin 653T

nuary 1977

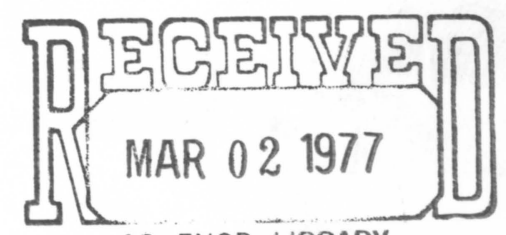

AG. ENGR. LIBRARY WEST VIRGINIA UNIVERSITY

est Virginia University

gricultural and Forestry Experiment Station

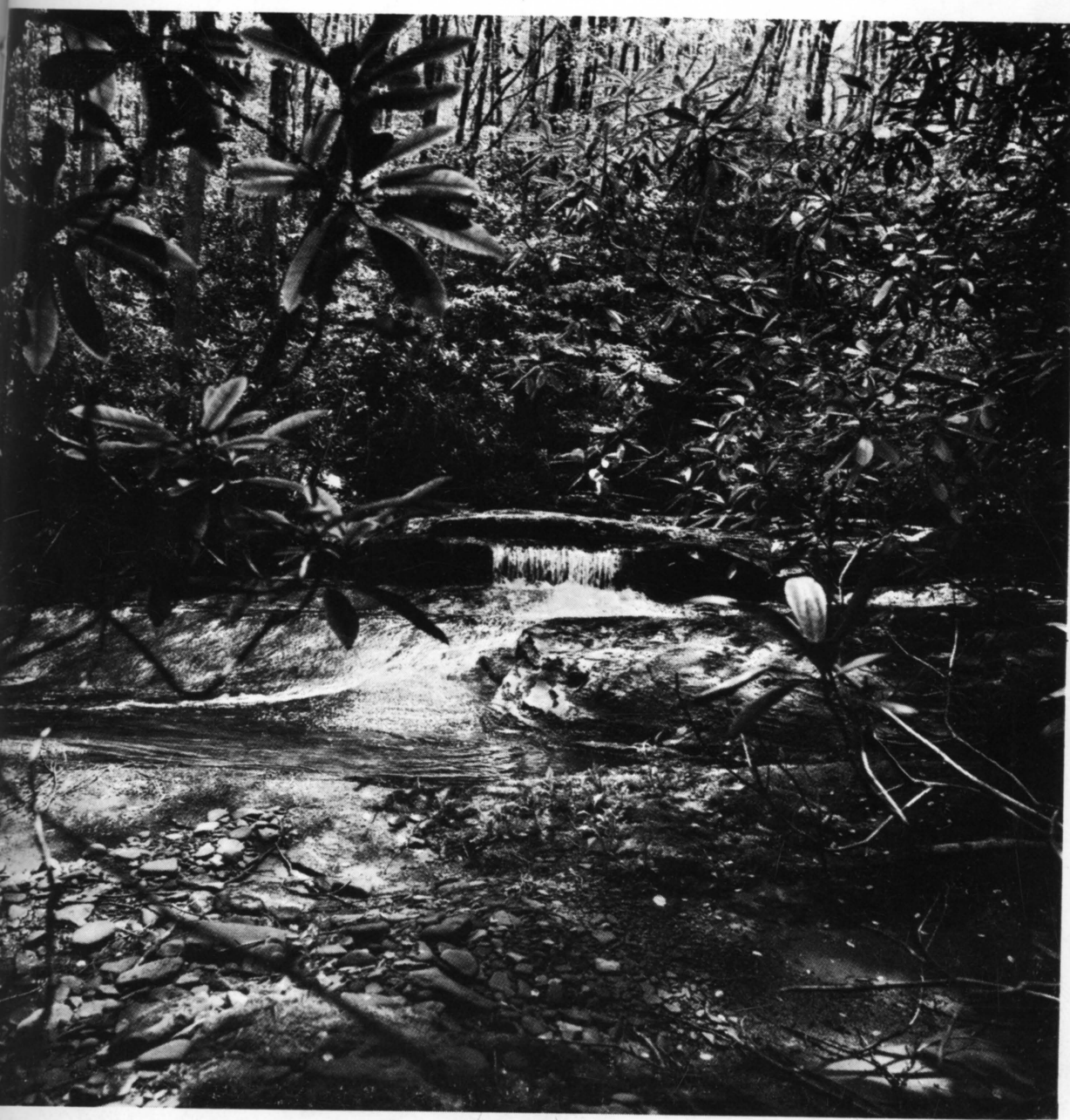


[Blank Page in Original Bulletin] 


\section{ABSTRACT}

A survey of 17 stations on nine northern West Virginia streams was conducted over a 12-month period to determine the effects of environmental quality on benthic aquatic insects. Monthly data included $\mathrm{pH}$, total acidity, dissolved oxygen, temperature, total iron, hardness, velocity and kinds and numbers of insects present. Benthic fauna reflected substratum, effects of weather and water quality.

All streams not receiving wastes were inhabited by numerous and diverse aquatic insects. Ephemera, Odonata and Diptera were predominant in sluggish streams with coarse sand beds. Swift, rocky bottomed streams yielded abundant Baetidae, Heptageniidae, Plecoptera, Trichoptera and various beetle and fly larvae. Streams subject to scouring during flash floods had low populations of insects.

A fertilizing effect accounting for increased numbers of insects was observed at a stream whose oxygen supply remained high in spite of mild organic enrichment. In a slow-moving, organically polluted stream with a fine sandy bed and lowered dissolved oxygen content the fauna was restricted to such forms as Hexagenia, Eristalis, Tipula and Chironomidae. Acid mine water supported sparse insect populations. Where water and rocks were colored by dense ferric hydroxide precipitates and $\mathrm{pH}$ averaged 3.3, only a few chironomid larvae (bloodworms) were found. However, in pools from a deep mine pumping station, these midge larvae were found in high numbers despite a pH of only 2.4 .

In general, aquatic insects respond in varying degrees to kinds and concentrations of wastes, and therefore, they serve as useful indicators of stream quality. 


\section{AUTHORS}

Bonnie Wojcik formerly was graduate student in entomology, Division of Plant Sciences; Linda Butler is Entomologist in the West Virginia University Agricultural and Forestry Experiment Station.

Indexing words: aquatic insects water pollution acid mine drainage environmental indicators

West Virginia University Agricultural and Forestry Experiment Station College of Agriculture and Forestry

Dale W. Zinn, Director

Morgantown 


\section{CONTENTS}

Methods and Materials $\ldots \ldots \ldots \ldots \ldots \ldots \ldots$

Description of Study Area $\ldots \ldots \ldots \ldots \ldots \ldots \ldots \ldots$

Results and Discussion $\ldots \ldots \ldots \ldots \ldots \ldots \ldots \ldots$

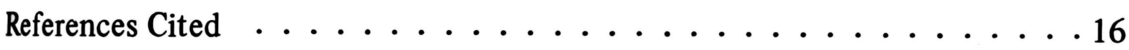

\section{TABLES}

1. Locality of stream sites sampled for aquatic insects $\ldots \ldots \ldots \ldots 2$

2. Chemical and physical data for streams for twelve months, from June, 1972, through May, 1973. Mean values and standard deviation are given for each parameter. . . . . . . . . . . . 5

3. Location of aquatic insect fauna in seventeen sampling sites (figures refer to index numbers listed in Table 1) from June, 1972, through May, 1973. . . . . . . . . . . . . . . 6

4. Total numbers of represented genera and individuals of aquatic insects collected at each site from June, 1972 through May, 1973 arranged according to water quality. $\ldots \ldots \ldots \ldots \ldots \ldots$

5. Environmental categories for sampling sites and associated aquatic insects. . . . . . . . . . . . . . . . 14 
[Blank Page in Original Bulletin] 


\section{Aquatic Insects as Indicators of Stream Environmental Quality in Northern West Virginia}

Expanding technology and man's rapidly increasing demand on environmental resources have necessitated new research on water quality and control of stream pollution. Practical, reliable methods for water analysis are being sought to help maintain streams sufficiently pure for human use and for aquatic life (Quigley, 1962). While chemical tests can reveal the exact nature of a pollutant, biological tests are more sensitive to mild or intermittent pollution and to substances which are overlooked in chemical analyses. Because of their convenient size, easy collection, lack of mobility, and varied tolerance to pollutants, aquatic insects are particularly useful as indicators of habitat quality (Goodnight, 1973).

Besides exhibiting the usual variety of physical characteristics, many northern West Virginia streams are affected by organic enrichment, sedimentation, and especially acid drainage from deep coal mines and strip mines.

In the present study an attempt was made to survey the aquatic insect fauna and to correlate the fauna qualitatively with environmental conditions in selected streams in northern West Virginia.

\section{METHODS AND MATERIALS}

Seventeen sampling stations on nine streams in a three-county region of northern West Virginia were chosen for study on the basis of accessibility, variety of waste input, and water depth (Table 1). Biological and chemical data were collected monthly from June, 1972 through May, 1973.

Aquatic insect communities were sampled in riffles by disturbing a two by four-foot region of the stream bed and catching the insects in a hand-held, 16-mesh screen as they floated downstream. Insects were killed and stored in 80 percent ethanol. Specimens were identified to genus, or family in some cases, according to Pennak (1953).

A YSI oxygen meter and probe (Model 51) were used to determine water temperature and dissolved oxygen content. The $\mathrm{pH}$ was determined from pHydrion control paper and a Corning 610-A expanded scale portable $\mathrm{pH}$ meter. Data for the first four months of study were unreliable because of a faulty $\mathrm{pH}$ meter. Stream velocity, recorded as linear meters per second at the water 


\begin{tabular}{|c|c|}
\hline Index No. & Locality \\
\hline 1. & $\begin{array}{l}\text { Aaron Creek (AC); } 0.4 \mathrm{~km} \text { from mouth; tributary of Decker's Creek, } \\
\text { Monongalia County. }\end{array}$ \\
\hline 2. & $\begin{array}{l}\text { Cobun Creek-station } 1\left(\mathrm{CC}_{1}\right) ; 2.0 \mathrm{~km} \text { from mouth; tributary of } \\
\text { Monongahela River, Monongalia County. }\end{array}$ \\
\hline 3. & Cobun Creek-station $2\left(\mathrm{CC}_{2}\right) ; 6.8 \mathrm{~km}$ from mouth. \\
\hline 4. & $\begin{array}{l}\text { White Day Creek-station } 1 \text { (WDC } 1) ; 2.3 \mathrm{~km} \text { from mouth; tributary of } \\
\text { Monongahela River, Monongalia County. }\end{array}$ \\
\hline 5. & White Day Creek-station $2\left(\mathrm{WDC}_{2}\right) ; 7.0 \mathrm{~km}$ from mouth. \\
\hline 6. & $\begin{array}{l}\text { Prickett's Creek-station } 1\left(\operatorname{PrC}_{1}\right) ; 3.6 \mathrm{~km} \text { from mouth; tributary of } \\
\text { Monongahela River, Marion County. }\end{array}$ \\
\hline 7. & Prickett's Creek-station $2\left(\mathrm{PrC}_{2}\right) ; 10.5 \mathrm{~km}$ from mouth. \\
\hline 8. & $\begin{array}{l}\text { Roaring Creek-station } 1\left(\mathrm{RC}_{1}\right) ; 0.3 \mathrm{~km} \text { from mouth; tributary of Cheat } \\
\text { River, Preston County. }\end{array}$ \\
\hline 9. & Roaring Creek-station $2\left(\mathrm{RC}_{2}\right) ; 10.1 \mathrm{~km}$ from mouth. \\
\hline 10. & $\begin{array}{l}\text { Little Lick Run (LLR); } 2.0 \mathrm{~km} \text { from mouth; tributary of Roaring Creek, } \\
\text { Preston County. }\end{array}$ \\
\hline 11. & $\begin{array}{l}\text { White's Run-station } 1\left(W_{1}\right) ; 0.4 \mathrm{~km} \text { from mouth; tributary of Lake } \\
\text { Lynn (Cheat Lake), Monongalia County. }\end{array}$ \\
\hline 12. & White's Run-station $2\left(\mathrm{WR}_{2}\right) ; 2.0 \mathrm{~km}$ from mouth. \\
\hline 13. & $\begin{array}{l}\text { Decker's Creek-station } 1\left(\mathrm{DC}_{1}\right) ; 17.7 \mathrm{~km} \text { from mouth; tributary of } \\
\text { Monongahela River, Monongalia County. }\end{array}$ \\
\hline 14. & Decker's Creek-station $2\left(\mathrm{DC}_{2}\right) ; 20 \mathrm{~km}$ from mouth. \\
\hline 15. & $\begin{array}{l}\text { Dent's Run-station } 1\left(\mathrm{DR}_{1}\right) ; 1.0 \mathrm{~km} \text { from mouth; tributary of } \\
\text { Monongahela River, Monongalia County. }\end{array}$ \\
\hline 16. & Dent's Run-station $2\left(\mathrm{DR}_{2}\right) ; 8.3 \mathrm{~km}$ from mouth. \\
\hline 17. & Dent's Run-station 3 (DR3); $12.5 \mathrm{~km}$ from mouth. \\
\hline
\end{tabular}

surface, was determined by the amount of time required for a float to traverse 7.6 meters. Water samples were carried to the laboratory, where hardness and acidity were determined by standard methods, and total iron was read from a Hach kit (Hach Chemical Company, Ames, Iowa) by the FerrOver method.

A survey of each stream was conducted to locate important tributaries and sources of wastes. Topographical maps aided in tracing the streams from origin to mouth, and in identifying towns and mines as possible sources of wastes. Qualitative benthic samples and pertinent chemical measurements of water quality were made at various locations along each stream and at some tributaries to determine specific waste sources.

\section{DESCRIPTION OF STUDY AREA}

All streams sampled in this study are in the Monongahela River drainage basin in the northern West Virginia counties of Monongalia, Preston and Marion 
(Table 1). The study area is characterized by dendritic stream systems which dissect unglaciated Appalachian Plateau land whose strata consist of shale, sndstone, limestone and coal beds (Friel et al., 1967; Doll et al., 1963).

Aaron Creek (AC). This lightly shaded stream is characterized by numerous iffles and a uniform stony bed which is interrupted along its $12.9-\mathrm{km}$ course only once by road construction, $2.8 \mathrm{~km}$ above the sampling site.

Cobun Creek (CC). Cobun Creek, which flows through $17.7 \mathrm{~km}$ of densely forested, lightly populated land, has fewer riffles than Aaron Creek, and the stones forming its bed are larger and more tightly packed. Two sampling stations were selected, one above and one below a section of road construction, the latter site being atypical of the stream because of its greater depth, sluggishness and easily scoured sandy bed.

White Day Creek (WDC). This stream with its numerous tributaries flows through largely undisturbed woodlands. The first sampling station has a rocky bed with patches of variously sized stones, while stones at site 2 , where the stream first flows from mountainous woodlands, are uniform in size. During heavy rains, White Day Creek attains a ruggedness unequalled by other study streams except possibly Decker's Creek.

Prickett's Creek (PrC). After passing through unpopulated woodlands for two-thirds its length, Prickett's Creek flows past several trailer parks and communities which contribute sewage to the stream. The bed consists of closely packed stones about $5-13 \mathrm{~cm}$ in diameter. The first sampling station was $0.8 \mathrm{~km}$ below sewage outfalls; site 2 was above them.

Roaring Creek (RC). This spring-fed creek flows through mountainous woodlands. Its lower half drains land on which strip mines and deep mines are located. Site 1, near the stream's mouth, is characterized by a patchy bed of variously sized stones colored red with ferric hydroxide from strip mines while station 2 lies above all sources of acid mine drainage. Except for the smaller overall size of the cobble, the bed at site 2 resembles that of the first site. This upper stream area is stocked with trout and fished in season.

Little Lick Run (LLR). At the sampling station this short, narrow tributary of Roaring Creek passes through a dairy farm, where the stream is about $0.5 \mathrm{~m}$ deep, sluggish and has a bed of fine sand and decomposing manure.

White's Run (WR) is only $4 \mathrm{~km}$ long, and at most $3.7 \mathrm{~m}$ wide and is the smallest stream sampled. At both study sites the stream bed consists of a patchy mixture of 5-8 cm flattened, irregular cobble, and variously sized pebbles.

Decker's Creek (DC) is the largest of the study streams and the one suffering the greatest number of disturbances. Near its source is a recent impoundment, beyond which the stream is dredged and widened for several kilometers, exposing a clay bottom, and producing an almost stagnant body of water. Several tributaries carrying sewage and acid water from deep mines enter Decker's Creek between the reservoir and the stream's mouth. A limestone 
company located $17.7 \mathrm{~km}$ from the mouth contributes large amounts of particulate limestone, which bury most of the stony bed in heavy silt and greatly reduces light penetration. One sampling station, site 1 , was located at the limestone company; the other site was above it at a place where the bed is composed largely of boulders.

Dent's Run (DR) flows undisturbed for about one-third its length providing a variety of aquatic habitats ranging from pools to riffles. Beyond this section the stream receives acid mine drainage from three tributaries which flow from four deep mine pumping stations. Three sampling stations were chosen for study, site 1, below all acid tributaries; site 2, between the first and second acid tributaries; and site 3, upstream from all acid mine drainage. Substrates consist of pebbles, small stones and silt at site 1 , moderate to large stones at site 2 , and coarse sand and pebbles less than $2.5 \mathrm{~cm}$ in diameter at site 3 .

\section{RESULTS AND DISCUSSION}

Chemical, physical, and biological data appear in Tables 2 and 3.

The following stream stations were free from point source waste input: both stations on $\mathrm{CC}, \mathrm{WR}$ and $\mathrm{WDC} ; \mathrm{RC}_{2}, \mathrm{PrC}_{2}, \mathrm{DR}_{3}$, and AC. Because no known pollutants affect the observed differences in quality and quantity of insect fauna found at these sites, an explanation of such differences lies in the variety of physical stream conditions encountered.

Stony beds of $\mathrm{RC}_{2}, \mathrm{WR}_{1}, \mathrm{WR}_{2}$, and $\mathrm{WDC}_{1}$ similarly consist of loose rubble and appear ideal as habitats for swift-water insects (Table 3 ). Table 4 reveals that these stream sections support large numbers of genera and individuals. Observed differences in total quantity of specimens may be due to chance, or they may reflect relative habitat suitabilities; e.g., $\mathrm{RC}$ is least prone to fluctuating velocity and volume of flow, WR maintains a relatively low flow, and WDC becomes swift following rains.

$\mathrm{AC}, \mathrm{PrC}_{2}$, and $\mathrm{WDC}_{2}$ are comparable to the previously discussed streams in number of genera, but the number of individuals is lower (Table 4). Because the substrate of these streams consists of closely laid larger stones instead of loose, variously sized ones, less between-stone living space is available, a fact which may account in part for the difference in numbers of insects found at these stations as compared to the number collected from sites such as $\mathrm{RC}_{2}$. A further point of difference may be the less frequent number of riffles at $\mathrm{PrC}_{2}$ and $\mathrm{WDC}_{2}$.

The lack of diversity and numbers of insects collected from CC is noteworthy (Tables 3 and 4). One explanation is the lower $\mathrm{pH}$ at these sites. Furthermore, the less-than-ideal substrates may be a contributing factor. The $\mathrm{CC}_{2}$ substratum consists of uniform, smooth rocks partially embedded in the earthen stream bottom. These stones are notably larger than those in the more productive streams. While $\mathrm{CC}_{1}$ lacks the embedded rock substratum, its sandy 
bed presents other problems. This area of the creek is particularly subject to flashflooding as shown by large quantities of debris on banks and in low-hanging tree branches after heavy rains. The scouring effect of flood waters on the sand bottom is severe.

$\mathrm{DR}_{3}$ seems to be an ideal habitat for those insects requiring minimal water flow and sandy substrate as shown by the high numbers of individuals collected (Table 4). The stream bed of coarse sand and small pebbles is easily colonized by burrowing insects such as Gomphidae, Ephemera and Chironomidae. While water levels rise considerably with heavy rainfall, effects of scouring are minimal.

The remaining seven collecting stations were contaminated with human and barnyard wastes $\left(\operatorname{PrC}_{1}, \mathrm{LLR}\right)$ or acid mine drainage $\left(\mathrm{RC}_{1}, \mathrm{DC}_{1}, \mathrm{DC}_{2}, \mathrm{DR}_{1}\right.$, and $\mathrm{DR}_{2}$ ).

An examination of Table 4 shows similar insect diversity for $\operatorname{PrC}_{1}$ and $\operatorname{PrC}_{2}$. However, the number of insects collected at $\mathrm{PrC}_{1}$ was more than twice that of $\mathrm{PrC}_{2}$. This abundance of benthic fauna at site 1 apparently resulted from organic

TABLE 2. CHEMICAL AND PHYSICAL DATA FOR STREAMS FOR TWELVE MONTHS, FROM JUNE, 1972, THROUGH MAY, 1973. MEAN VALUES AND STANDARD DEVIATION ARE GIVEN FOR EACH PARAMETER.

\begin{tabular}{|c|c|c|c|c|c|}
\hline $\begin{array}{l}\text { Sampling } \\
\text { Station }\end{array}$ & $\mathrm{pH}^{1}$ & $\begin{array}{l}\text { Total Iron } \\
\text { (ppm) }\end{array}$ & $\begin{array}{c}\text { Total Acid } 2 \\
\left(\mathrm{Mg} / \mathrm{L} \mathrm{CaCO}_{3}\right)\end{array}$ & $\begin{array}{l}\text { Total Hardness } \\
\text { (Mg/L CaCo3) }\end{array}$ & $\begin{array}{c}\text { Velocity } \\
\text { (meters/sec) }\end{array}$ \\
\hline $\mathrm{AC}$ & $5.7 \pm 0.7$ & $0.2 \pm 0.2$ & - & $80 \pm 25$ & $0.823 \pm 0.396$ \\
\hline $\mathrm{CC}_{1}$ & $5.3 \pm 0.8$ & $0.1 \pm 0.1$ & - & $92 \pm 26$ & $0.396 \pm 0.183$ \\
\hline $\mathrm{CC}_{2}$ & $5.1 \pm 0.5$ & $0.1 \pm 0.1$ & - & $60 \pm 19$ & $0.518 \pm 0.244$ \\
\hline $\mathrm{WDC}_{1}$ & $5.9 \pm 0.3$ & $0.1 \pm 0.1$ & - & $49 \pm 17$ & $0.518 \pm 0.274$ \\
\hline $\mathrm{WDC}_{2}$ & $5.7 \pm 0.3$ & $0.1 \pm 0.2$ & - & $40 \pm 10$ & $0.640 \pm 0.396$ \\
\hline $\mathrm{PrC}_{1}$ & $5.6 \pm 0.3$ & $0.1 \pm 0.1$ & - & $64 \pm 29$ & $0.732 \pm 0.366$ \\
\hline $\mathrm{PrC}_{2}$ & $5.4 \pm 0.3$ & $0.1 \pm 0.1$ & - & $39 \pm 11$ & $0.549 \pm 0.335$ \\
\hline $\mathrm{RC}_{1}$ & $3.6 \pm 0.2$ & $0.8 \pm 0.3$ & $29 \pm 18$ & $125 \pm 54$ & $0.488 \pm 0.244$ \\
\hline $\mathrm{RC}_{2}$ & $5.4 \pm 0.2$ & $0.1 \pm 0.2$ & - & $38 \pm 11$ & $0.671 \pm 0.213$ \\
\hline LLR & $5.2 \pm 0.4$ & $0.3 \pm 0.3$ & - & $38 \pm 8$ & $0.152 \pm 0.061$ \\
\hline $\mathrm{WR}_{1}$ & $5.9 \pm 0.5$ & $0.2 \pm 0.1$ & - & $202 \pm 64$ & $0.396 \pm 0.244$ \\
\hline $\mathrm{WR}_{2}$ & $6.0 \pm 0.6$ & $0.2 \pm 0.1$ & $8 \pm$ & $400 \pm 242$ & $0.427 \pm 0.427$ \\
\hline $\mathrm{DC}_{1}$ & $3.7 \pm 0.4$ & $1.5 \pm 0.8$ & $18 \pm 8$ & $178 \pm 96$ & $0.518 \pm 0244$ \\
\hline $\mathrm{DC}_{2}$ & $3.4 \pm 0.5$ & $2.4 \pm 0.7$ & $57 \pm 42$ & $136 \pm 66$ & $0.549 \pm 0.274$ \\
\hline $\mathrm{DR}_{1}$ & $3.3 \pm 0.5$ & $48.7 \pm 19.9$ & $359 \pm 415$ & $528 \pm 322$ & $0.579 \pm 0.213$ \\
\hline $\mathrm{DR}_{2}$ & $3.6 \pm 0.5$ & $59.2 \pm 19.5$ & $208 \pm 260$ & $441 \pm 254$ & $0.518 \pm 0.152$ \\
\hline $\mathrm{DR}_{3}$ & $6.6 \pm 0.6$ & $0.1 \pm 0.1$ & $=$ & $173 \pm 35$ & $0.152 \pm 0.152$ \\
\hline
\end{tabular}

${ }_{1}$ While values for all other parameters represent means of 12 sampling periods, that for $\mathrm{pH}$ is for 8 sampling periods only, October 1972-May, 1973.

$2 \mathrm{~A}$ dash (-) indicates only a trace amount. 
TABLE 3. LOCATION OF AQUATIC INSECT FAUNA IN SEVENTEEN SAMPLING SITES (FIGURES REFER TO INDEX NUMBERS LISTED IN TABLE 1) FROM JUNE, 1972, THROUGH MAY, 1973.

\begin{tabular}{|c|c|c|c|c|c|c|c|c|c|c|c|c|}
\hline Taxa & June & July & Aug. & Sept. & Oct. & Nov. & Dec. & Jan. & Feb. & Mar. & Apr. & May \\
\hline $\begin{array}{l}\text { Ephemeroptera- } \\
\text { Hexagenia }\end{array}$ & 10 & & 10 & & & & 10 & & & & & 10 \\
\hline Ephemera & 17 & 6,17 & 17 & 17 & $2,5,17$ & 6,17 & $\begin{array}{l}1,2,5 \\
17\end{array}$ & 2,17 & 4,7 & 2,17 & $\begin{array}{l}2,3,5, \\
17\end{array}$ & $5,9,17$ \\
\hline Baetisca & & & & & & & & & & 2 & 2 & \\
\hline Ephemerella & $\begin{array}{l}4,5,6 \\
7,8,9 \\
11,12 \\
17\end{array}$ & 9,11 & 9 & & 6 & & 17 & 1,9 & 9 & 9 & $\begin{array}{l}4,5,7 \\
9,11 \\
17\end{array}$ & $\begin{array}{l}4,5,6 \\
7,8,9 \\
10,11\end{array}$ \\
\hline Blasturus & & & & & & & & & & 10 & & \\
\hline Paraleptophlebia & $5,6,7$ & & & 6 & & & & & & 5,7 & & 11 \\
\hline Baetis & & & & & & & & & 9 & & & 9 \\
\hline Ameletus & $\begin{array}{l}6,7,9 \\
11,12\end{array}$ & 5 & 1,11 & $\begin{array}{l}2,3,7 \\
11\end{array}$ & $1,9,11$ & 3 & & 9 & 9 & $\begin{array}{l}1,4,5 \\
6,7,9\end{array}$ & $\begin{array}{l}4,5,6, \\
7,9,11, \\
12\end{array}$ & $\begin{array}{l}4,6,7, \\
9,11, \\
12\end{array}$ \\
\hline Siphlonurus & 10 & & & & & & & & & & & \\
\hline Isonychia & 4,6 & $4,5,6$ & $\begin{array}{l}1,4,5 \\
6\end{array}$ & $\begin{array}{l}1,4,6 \\
7\end{array}$ & $4,6,7$ & $\begin{array}{l}3,4,5 \\
6\end{array}$ & 6 & 5 & & 4 & 4 & $1,4,5$ \\
\hline Iron & $4,9,11$ & & & & & & & 9 & & 7,9 & 9 & 6,9 \\
\hline Heptagenia & $\begin{array}{l}4,6,7, \\
9,11, \\
12\end{array}$ & $4,5,6$ & 1,2 & & & & & & & 9 & 9 & $\begin{array}{l}4,5,7 \\
9,11\end{array}$ \\
\hline
\end{tabular}


(Table 3 continued)

\begin{tabular}{|c|c|c|c|c|c|c|c|c|c|c|c|c|}
\hline Taxa & June & July & Aug. & Sept. & Oct. & Nov. & Dec. & Jan. & Feb. & Mar. & Apr. & May \\
\hline Stenonema & $\begin{array}{l}1,2,4, \\
5,6,7, \\
11\end{array}$ & $\begin{array}{l}2,4,5 \\
6,11, \\
12\end{array}$ & $\begin{array}{l}1,4,6 \\
9,11, \\
12,17\end{array}$ & $\begin{array}{l}1,2,4 \\
6,7,9 \\
11\end{array}$ & $\begin{array}{l}1,2,3 \\
4,5,6, \\
7,9,17\end{array}$ & $\begin{array}{l}1,2,3 \\
4,5,6 \\
9\end{array}$ & $\begin{array}{l}1,4,5 \\
6\end{array}$ & $\begin{array}{l}1,5,6 \\
7,9\end{array}$ & $\begin{array}{l}1,4,5 \\
7,11\end{array}$ & $\begin{array}{l}1,2,3 \\
5,6,7 \\
11\end{array}$ & $\begin{array}{l}1,2,4 \\
5,6,7 \\
9,11\end{array}$ & $\begin{array}{l}1,4,6 \\
9,11\end{array}$ \\
\hline \multicolumn{13}{|l|}{ Plecoptera- } \\
\hline Pteronarcys & & 5 & 9 & 5,9 & 5,9 & 5 & 5 & 7 & & 9 & 7,9 & 5,9 \\
\hline Peltoperla & & & & 9 & 9 & 9 & & 9 & & 9 & 9 & \\
\hline Nemoura & 12 & & & & & & & & & & $\begin{array}{l}11,12, \\
17\end{array}$ & $\begin{array}{l}1,4,9 \\
11,12\end{array}$ \\
\hline Brachyptera & & & & & & & & 12 & & $4,6,7$ & 11,12 & \\
\hline Taeniopteryx & & & & & & & 5 & 6 & 5 & 4 & & \\
\hline Allocapnia & & & & & & & 5,6 & 1,12 & & & & \\
\hline Isoperla & $\begin{array}{l}4,9,11, \\
12\end{array}$ & & & 9 & & 9 & 9 & $\begin{array}{l}9,11 \\
12\end{array}$ & $\begin{array}{l}9,11 \\
12\end{array}$ & 9,11 & $\begin{array}{l}9,11 \\
12,17\end{array}$ & $\begin{array}{l}3,4,6, \\
7,8,11, \\
12\end{array}$ \\
\hline Isogenus & 12 & & & & & & & 12 & & & 12 & \\
\hline Acroneuria & $\begin{array}{l}1,2,4, \\
6,7,9, \\
11,12\end{array}$ & $\begin{array}{l}5,9,11 \\
12,17\end{array}$ & $\begin{array}{l}2,4,6 \\
9,12\end{array}$ & $\begin{array}{l}2,4,5 \\
7,9\end{array}$ & $\begin{array}{l}3,4,6 \\
7,12\end{array}$ & $\begin{array}{l}3,4,5 \\
6\end{array}$ & 4,7 & 5,12 & $\begin{array}{l}3,5,9 \\
12\end{array}$ & $\begin{array}{l}1,5,7 \\
9\end{array}$ & $\begin{array}{l}3,4,5 \\
9,12\end{array}$ & $\begin{array}{l}5,7,11, \\
12\end{array}$ \\
\hline Neoperla & & & & 12 & & & 1 & & & 1 & & \\
\hline Perlesta & 4 & & & & & & & & & & & 4 \\
\hline Alloperla & & & & & & & & & & & & 7 \\
\hline \multicolumn{13}{|l|}{ Odonata- } \\
\hline Cordulegaster & 12,17 & 7,17 & 10 & 17 & 10 & 7,12 & 7 & & & 17 & 17 & 17 \\
\hline $\begin{array}{l}\text { Libellula } \\
\text { Boyeria }\end{array}$ & & & & $\begin{array}{l}17 \\
11.17\end{array}$ & 17 & & & & & 5 & 5 & \\
\hline
\end{tabular}


(Table 3 continued)

\begin{tabular}{|c|c|c|c|c|c|c|c|c|c|c|c|c|}
\hline Taxa & June & July & Aug. & Sept. & Oct. & Nov. & Dec. & Jan. & Feb. & Mar. & Apr. & May \\
\hline Gomphus & & & & & 17 & & & & & & & \\
\hline Lanthus & 7 & 7 & 9 & 9,17 & $\begin{array}{l}2,5,6 \\
7,9,11\end{array}$ & $6,7,12$ & 1,17 & 9 & 7 & $\begin{array}{l}1,5,9, \\
17\end{array}$ & 17 & $1,5,17$ \\
\hline Dromogomphus & 17 & & & 17 & & & & & & & & \\
\hline Calopteryx & & & & 11,17 & $\begin{array}{l}1,2,12, \\
17\end{array}$ & 12 & & & & & & \\
\hline Coenagrionidae & & & & & 6 & & & & & & & \\
\hline Neuroptera- & & & & & & & & & & & & \\
\hline Sialis & & 11 & & 12 & 8,17 & 8 & 1 & $1,5,14$ & & & & 8 \\
\hline Corydalus & $4,6,7$ & 4,6 & $2,4,6$ & 4,6 & $1,4,6$ & $4,5,6$ & 1,2 & & & 1 & 4 & 4,6 \\
\hline $\begin{array}{l}\text { Nigronia } \\
\text { Trichoptera- }\end{array}$ & $\begin{array}{l}1,2,5 \\
9,11\end{array}$ & $5,11,12$ & 2,17 & $\begin{array}{l}1,3,4, \\
11\end{array}$ & 3,5 & 5 & 1 & 5 & 5 & $\begin{array}{l}10,11, \\
12\end{array}$ & 5,7 & 11 \\
\hline Helicopsyche & & 1,7 & & 5,7 & & & & & 1 & 1 & 2 & 6 \\
\hline Hydropsyche & $\begin{array}{l}2,4,6 \\
7,9,11\end{array}$ & $\begin{array}{l}1,3,5 \\
6,11\end{array}$ & $\begin{array}{l}2,4,6 \\
7,11\end{array}$ & $\begin{array}{l}1,3,4 \\
5,6,7 \\
9,11\end{array}$ & $\begin{array}{l}1,2,4 \\
6,7,11\end{array}$ & $\begin{array}{l}1,3,4 \\
5,6,9\end{array}$ & $\begin{array}{l}1,3,6, \\
9\end{array}$ & $\begin{array}{l}1,7,9 \\
11\end{array}$ & 1,11 & $\begin{array}{l}1,4,6, \\
9\end{array}$ & $\begin{array}{l}1,3,5 \\
6,7,9 \\
11\end{array}$ & $\begin{array}{l}3,4,6 \\
9,11\end{array}$ \\
\hline Cheumatopsyche & $\begin{array}{l}3,4,6, \\
7,11, \\
12\end{array}$ & $\begin{array}{l}5,6,11, \\
12\end{array}$ & $\begin{array}{l}2,4,6, \\
7,11 \\
12\end{array}$ & $\begin{array}{l}1,2,3 \\
4,5,6 \\
7,11\end{array}$ & $\begin{array}{l}1,2,3, \\
4,5,6, \\
7,9,11, \\
12,17\end{array}$ & $\begin{array}{l}1,3,4, \\
5,6,9 \\
17\end{array}$ & $1,6,12$ & $\begin{array}{l}1,6,9 \\
11\end{array}$ & $\begin{array}{l}1,6,11, \\
12\end{array}$ & $\begin{array}{l}1,3,6, \\
7,9,12\end{array}$ & $\begin{array}{l}1,5,6, \\
9,11,12\end{array}$ & 6 \\
\hline Diplectrona & $\begin{array}{l}3,6,8 \\
9,12\end{array}$ & $9,11,12$ & $9,11,12$ & 9 & & & 3,12 & 9 & 12 & 9,12 & $9,11,12$ & 9,12 \\
\hline $\begin{array}{l}\text { Rhyacophila } \\
\text { Glossosoma }\end{array}$ & 9 & 9 & & & & & & 9 & & 12 & $\begin{array}{l}7,11 \\
9\end{array}$ & 6 \\
\hline
\end{tabular}


(Table 3 continued)

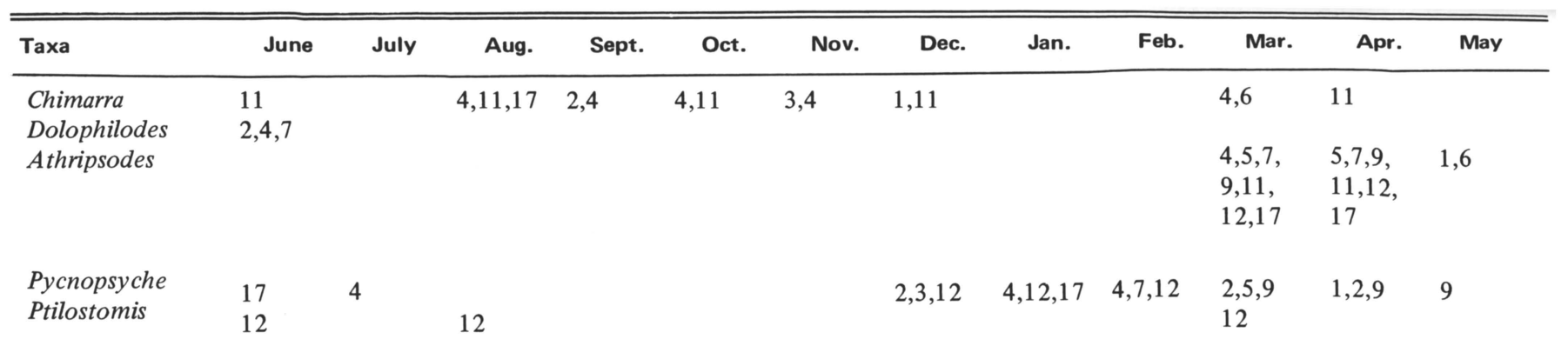

Coleoptera-

Hydrophilidae 12

Dytiscidae

12

Psephenida'

$1,2,3$

\begin{tabular}{lllll} 
& \multicolumn{3}{c}{17} & \\
$6,7,11$ & $2,6,7$, & $3,6,11$ & $2,6,11$ & 6
\end{tabular}

$\begin{array}{lll} & & 8 \\ 1,7 & 11 & 1,6\end{array}$

$1,6,7, \quad 6,11$

6,11

Elmidae/ 6,7

11,12

\begin{tabular}{|c|c|c|c|c|}
\hline $2,6,11$ & 7 & $\begin{array}{l}1,2,4, \\
11,12\end{array}$ & $\begin{array}{l}2,3,6 \\
7,8,9 \\
11,17\end{array}$ & $\begin{array}{l}1,4,7 \\
9,11\end{array}$ \\
\hline
\end{tabular}

$1,12 \quad 1$

1

$1,3,6, \quad 1,11$

Diptera-

Tipula

$1,7,12$

$\begin{array}{lll}2,10, & 2,3,6, & 2,6,7 \\ 12,17 & 9,11,17 & 12,17\end{array}$

$1,2,5, \quad 1,3,5, \quad 1,2,3, \quad 2,3,4, \quad 1,4,8$,

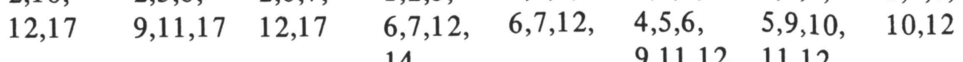
$14 \quad 9,11,12,11,12$

17

Antocha

Pedicia 
(Table 3 continued)

\begin{tabular}{|c|c|c|c|c|c|c|c|c|c|c|c|c|}
\hline Taxa & June & July & Aug. & Sept. & Oct. & Nov. & Dec. & Jan. & Feb. & Mar. & Apr. & May \\
\hline Hexatoma & 2,6 & & 7,10 & 7,17 & $\begin{array}{l}1,2,7 \\
17\end{array}$ & 1,2 & 17 & 1,7 & & $\begin{array}{l}1,2,7 \\
9,17\end{array}$ & $\begin{array}{l}1,2,7 \\
9,12,17\end{array}$ & $\begin{array}{l}1,4,6 \\
7,9,17\end{array}$ \\
\hline Blepharocera & 9 & & & & & & & & & & & 9 \\
\hline Atherix & & & & 7 & 7 & 4,7 & & 7 & & $5,6,9$ & 6 & \\
\hline $\begin{array}{l}\text { Chrysops } \\
\text { Eristalis }\end{array}$ & 10 & 6,17 & & 10,17 & 10,17 & & 17 & 17 & & 9 & & \\
\hline Limnophora & & & 6,7 & & & & & & & 5 & & \\
\hline Culicidae & 5 & & 10 & & & & & & & & & \\
\hline Simuliidae & & & 11 & & & & & & & & & \\
\hline Chironomidae & 11,17 & $\begin{array}{l}10,13 \\
17\end{array}$ & $\begin{array}{l}9,10 \\
14\end{array}$ & $\begin{array}{l}7,8,10 \\
14,17\end{array}$ & $\begin{array}{l}8,9,10 \\
15,16 \\
17\end{array}$ & $\begin{array}{l}2,8,10 \\
15,17\end{array}$ & $\begin{array}{l}2,4,5 \\
6,14, \\
17\end{array}$ & $\begin{array}{l}2,10 \\
14,17\end{array}$ & 10 & $\begin{array}{l}6,9,10, \\
11,12 \\
17\end{array}$ & $\begin{array}{l}1,4,5 \\
6,9,12, \\
17\end{array}$ & $\begin{array}{l}1,3,4, \\
6,9,11 \\
12,17\end{array}$ \\
\hline
\end{tabular}


TABLE 4. TOTAL NUMBERS OF REPRESENTED GENERA AND INDIVIDUALS OF AQUATIC INSECTS COLLECTED AT EACH SITE FROM JUNE, 1972 THROUGH MAY, 1973 ARRANGED ACCORDING TO WATER QUALITY.

\begin{tabular}{|c|c|c|}
\hline Site & No. Represented Genera & Total Individuals \\
\hline \multicolumn{3}{|c|}{ No Known Wastes } \\
\hline $\mathrm{DR}_{3}$ & 25 & 712 \\
\hline $\mathrm{RC}_{2}$ & 28 & 583 \\
\hline $\mathrm{WR}_{2}$ & 29 & 518 \\
\hline $\mathrm{WR}_{1}$ & 26 & 474 \\
\hline $\mathrm{WDC}_{1}$ & 26 & 472 \\
\hline $\mathrm{AC}$ & 27 & 395 \\
\hline $\mathrm{PrC}_{2}$ & 32 & 305 \\
\hline $\mathrm{CC}_{1}$ & 21 & 241 \\
\hline $\mathrm{WDC}_{2}$ & 27 & 235 \\
\hline $\mathrm{CC}_{2}$ & 16 & 85 \\
\hline \multicolumn{3}{|l|}{ Drganic Wastes } \\
\hline $\mathrm{PrC}_{1}$ & 32 & 703 \\
\hline LLR & 13 & 104 \\
\hline \multicolumn{3}{|c|}{ Acid Mine Water } \\
\hline $\mathrm{RC}_{1}$ & 8 & 36 \\
\hline $\mathrm{DC}_{2}$ & 3 & 64 \\
\hline $\mathrm{DR}_{1}$ & 1 & 16 \\
\hline $\mathrm{DR}_{2}$ & 1 & 1 \\
\hline $\mathrm{DC}_{1}$ & 1 & 1 \\
\hline
\end{tabular}

enrichment of the stream by sewage entering upstream. Net-spinning trichopterans were presented in especially large numbers and many possessed growths which strongly resembled Epistylis, a "sewage fungus" known to thrive where organic content is high and oxygen is plentiful (Hynes, 1963).

Compared to all streams discussed above, LLR was notably lacking in diversity and total numbers of aquatic insects (Table 4). Several factors contribute to this effect. The bed of fine, shifting sand and organics from decomposing manure is of a type known to have poor fauna (Hynes, 1970). Since this stream site is utilized as a watering area for pastured cattle, their disrupting effect cannot be overlooked. Periodic drops in concentration of dissovled oxygen in this sluggish, organically enriched stream undoubtedly limited some species. Hexagenia, which was collected solely at LLR, is known to survive at very low $\mathrm{O}_{2}$ levels (Eriksen, 1964). Additionally, the air-breathing Eristalis, Tipula and hemoglobin-containing chironomids are specially adapted for life in such an environment. 
While the environment at $\mathrm{RC}_{1}$ is not as hazardous to aquatic fauna as that at the four remaining sites, diversity and numbers of insects were low. This site would appear to be a suitable habitat were it not for the consistently low $\mathrm{pH}$ from acid mine drainage. Chironomids and Sialis were collected on several occasions. Both animals are known to be relatively tolerant of acid conditions (Weed and Rutschky, 1972; Koryak et al., 1972). The collection of Ephemerella, Isoperla and Diplectrona in May and June is noteworthy. While they were not abundant, all genera were collected regularly in repeated samplings on individual collection days. Appearance of acid intolerant species was related to high rainfall. Heavy spring rains increased stream volume, washing benthic forms from suitable upstream habitats near $\mathrm{RC}_{2}$. Temporary dilution of the acid allowed survival of these insects at $\mathrm{RC}_{1}$.

$\mathrm{DR}_{1}$ and $\mathrm{DR}_{2}$, which regularly receive acid mine drainage, had low $\mathrm{pH}$ values and high concentrations of iron (Table 2). Otherwise biologically suitable substrates are coated with thick orange deposits of ferric hydroxide; even the water is orange. A total of 17 chironomids was found at both sites during the year. Low species diversity and quantity of individuals are characteristic of conditions of high acidity, iron, sulfate and buildup of sediments (Koryak et al. 1972).

Effects of acid mine drainage on aquatic life were seen vividly in the DR watershed. It was noted earlier that upper $\mathrm{DR}$ in the area of our $\mathrm{DR}_{3}$ showec great diversity and abundance of insects. For $1.2 \mathrm{~km}$ downstream from $\mathrm{DR}_{3}$ fish, crustaceans, and all orders of aquatic insects were bountiful. At that point however, the first acid tributary ( $\mathrm{pH} 2.3$ ) created by a deep-mine pumping station, enters DR. Aquatic fauna were observed living to the boundary of the incoming mine water. while none were found downstream from the boundary.

In tracing the various acid mine tributaries of DR, our observations confirmed the contention of Koryak et al., (1972) that highly acid portions of streams may exhibit "low diversity (even as low as a single species) with high numbers of individuals." A highly acid pool of $\mathrm{pH} 2.4$ on the third acid tributary was found to have a high population of chironomid larvae.

In a survey of the $40^{+} \mathrm{km}$ of DC, numerous disturbances were located including impoundment, channelization, sewage and acid mine drainage. Beginning with a reservoir near its source these disturbances have rendered most of the stream virtually devoid of aquatic insects. Samplings from stones at the reservoir spillway produced several trichopterans. Below the spillway, the stream passes through cultivated farmland. Samples from the sandy bed resulted in sparse numbers of tipulids and chironomids. An impoverished state of stream fauna below impoundments has been related to siltation, increased concentra tion of phosphorus and nitrogen or altered thermal regimes (Hilsenhoff, 1971 Lehmkuhl, 1972). 
Downstream from the area of siltation, DC has been widened and channelized by dredging. The bed of hard clay or fine sand is an unsuitaile habitat. At this point a small tributary bearing raw sewage and another with acid mine drainage enter the creek. For the next several kilometers, DC maintains a $\mathrm{pH}$ of approximately 4.0-4.2. Only corixids were found in several samples. Between this point and the monthly sampling site, $\mathrm{DC}_{2}$, approximately $10 \mathrm{~km}$ lownstream, are additional influxes of sewage and acid mine drainage so that the iH level is reduced to an average 3.4. Ferric hydroxide deposits coat the stones. Jespite the almost prohibitive $\mathrm{pH}$, swift water and boulder-like substrate, iccasional tipulids and Sialis were found. Particularly notable was a mass mergence of chironomids in late summer.

$\mathrm{DC}_{1}$, located downstream at a limestone company, was a considerably less desirable habitat. Fine particulate limestone carried in the water blocks the passage of light and when settling out, buries the stream bottom in a heavy layer of sediment. Even chironomids were unable to tolerate this harsh environment. Despite a steady rise in $\mathrm{pH}$ up to 4.8 downstream from $\mathrm{DC}_{1}$ no aquatic life was collected.

Several physical and chemical parameters which were monitored during the course of this study showed varying degrees of importance with reference to insect fauna.

A summary of stream characteristics and associated indicator insects is given in Table 5. Temperature was apparently not a limiting factor at any of the sampling sites. Most streams showed predictable seasonal variation, ranging from near $0^{\circ} \mathrm{C}$ in the winter to about $22^{\circ} \mathrm{C}$ in late summer. A notable exception was spring-fed RC which exhibited a narrower temperature range with season. With the possible exception of LLR, oxygen deficiency did not appear to be a limiting factor at any of the study sites.

The total hardness (Table 2) measured in this study was caused by divalent metallic cations. Hardness alone cannot be considered a limiting factor in this study. Most of the sampling sites showing water to be moderately hard (75 - 150 $\left.\mathrm{mg} / 1 \mathrm{CaCO}_{3}\right)$ to very hard $\left(300+\mathrm{mg} / 1 \mathrm{CaCO}_{3}\right)$ (according to the classification of Sawyer and McCarty, 1967) were those sites receiving acid mine drainage $\left(\mathrm{RC}_{1}, \mathrm{DR}_{1}, \mathrm{DR}_{2}, \mathrm{DC}\right)$. Generally, other streams were soft. Notable exceptions were $\mathrm{DR}_{3}$ and WR, which have total hardness levels equal to some of the acid ladened streams but which support a large and diverse fauna.

Many streams in northern West Virginia carry acid mine water which has high concentrations of iron. It is well known that such streams support little life (Hynes, 1963), although it may be difficult to separate the related effects of reduced light penetration, sedimentation and low $\mathrm{pH}$. In the present study, streams having iron concentrations as low as $0.8 \mathrm{ppm}$ supported low numbers and kinds of organisms (Table 5). 


\section{TABLE 5. ENVIRONMENTAL CATEGORIES FOR SAMPLING SITES AND ASSOCIATED AQUATIC INSECTS.}

\begin{tabular}{ccc}
\hline \hline Stream & & \\
Sampling & Important Physical and & Indicator Insects \\
Station (s) & Chemical Characteristics & A \\
\hline
\end{tabular}

\section{I. $\mathrm{DR}_{3}$ \\ Sluggish Streams, Sandy Beds, Numerous Pools \\ Well-oxygenated, bed of coarse sand and gravel (less than $1 \mathrm{~cm}$ diameter); no wastes entering water; minimal scouring during floods; $\mathrm{pH}$ near neutral.}

II. $\mathrm{CC}_{1}$

III. LLR

Poorly oxygenated; bed of fine sand
Similar to I above, except $\mathrm{pH}$ drops lower; much scouring occurs during flash floods. and decomposing manure.
Numbers and diversity of insects high; burrowing insects prevalentEmphemera, Cordulegasteridae, Gomphidae, Tipula, Chironomidae.

Similar to I, but with lower numbers and diversity.

Still lower numbers and diversity of insects; burrowers and air breathers prevalent-Hexagenia, Eristalis, Tipula, Chrysops, Chironomidae.

\section{Well-Oxygenated, Shallow, Non-Sluggish Streams with Rocky Beds}

IV. $\mathrm{RC}_{2}$ Numerous riffles, loose

$\mathrm{WR}_{1}$ stones of assorted sizes; no

$\mathrm{WR}_{2}$ wastes entering water. $\mathrm{WDC}_{1}$

AC

V. $\mathrm{WDC}_{2} \quad$ Few riffles; closely packed stones $\mathrm{PrC}_{2}$ of uniform size (7-10 cm diameter). No known wastes entering water.

VI. $\quad \mathrm{CC}_{2} \quad$ Similar to $\mathrm{V}$, but with stones generally larger than $10 \mathrm{~cm}$ diameter; $\mathrm{pH}$ occasionally drops.

VII. $\operatorname{PrC}_{1}$ Numerous riffles, loose stones of assorted sizes; mild organic enrichment.

VIII. $\mathrm{RC}_{1}$ Numerous riffles, loose stones of assorted sizes; acid mine drainage present. Iron $=0.8 \mathrm{ppm}$; average $\mathrm{pH}=3.6$; little $\mathrm{Fe}(\mathrm{OH})_{3}$ precipitated on stones.
Aquatic insects of all relevant orders present; prevalence of types adapted for swift water and stony bed-Heptageniidae, Baetidae, Psephenus, Blepharocera, Plecoptera, Trichoptera.

Similar to IV above, but lower in numbers of specimens.

All aquatic orders represented but diversity and total numbers much lower than in V.

Diversity high, quantity very high. Hydropsychidae common.

Prevalence of swift-water insects.

Greatly restricted quantity and diversity. Sialis and Chironomidae present. Few Diptera, Plecoptera, Ephemeroptera or Trichoptera. 

ASSOCIATED AQUATIC INSECTS.

\begin{tabular}{|c|c|c|c|}
\hline \multicolumn{2}{|c|}{$\begin{array}{l}\text { Stream } \\
\text { Sampling } \\
\text { Station (s) }\end{array}$} & \multirow[b]{2}{*}{$\begin{array}{l}\text { Similar to VIII, but substrate } \\
\text { of very large rock only; iron }= \\
2.4 \mathrm{ppm} \text {. }\end{array}$} & Indicator Insects \\
\hline IX. & $\mathrm{DC}_{2}$ & & $\begin{array}{l}\text { Chironomidae and Sialis present } \\
\text { but uncommon. }\end{array}$ \\
\hline $\mathrm{X}$. & $\begin{array}{l}\mathrm{DR}_{1} \\
\mathrm{DR}_{2}\end{array}$ & $\begin{array}{l}\text { Numerous riffles, loose stones of } \\
\text { various sizes; acid mine drainage } \\
\text { present. Iron }=40-60 \text { ppm; average } \\
\text { pH }=3.3-3.6 \text {; stones heavily coated } \\
\text { with } \mathrm{Fe}(\mathrm{OH})_{3} \text {; water orange. }\end{array}$ & $\begin{array}{l}\text { Chironomidae only; even these } \\
\text { are rare. }\end{array}$ \\
\hline XI. & $\mathrm{DC}_{1}$ & $\begin{array}{l}\text { Water polluted with acid mine drain- } \\
\text { age and limestone sediment; average } \\
\mathrm{pH}=3.7 \text {; light does not penetrate } \\
\text { to bed; stones nearly covered by } \\
\text { heavy limestone sediment. }\end{array}$ & Same as $\mathbf{X}$. \\
\hline
\end{tabular}

Insignificant levels of acidity were recorded except for those streams receiving acid mine drainage (Table 2). While all these sites showed similar $\mathrm{pH}$ levels 3.3-3.7, total acidity was markedly different, being four to six times higher for $\mathrm{DR}_{1}$ and $\mathrm{DR}_{2}$ than for $\mathrm{RC}_{1}, \mathrm{DC}_{1}$ and $\mathrm{DC}_{2}$. The stress placed on aquatic organisms by acid mine drainage is evidenced by the virtual absence of benthic macroinvertebrates in affected streams. Chironomid midge larvae (bloodworms) were the most abundant insects in acid waters in this study (Tables 3,5 ).

Water velocity, while an important factor in determining kinds and numbers of aquatic insects, perhaps should be considered together with substrate differences. In this study, stream sites with the lowest water velocity $\left(D_{3}\right.$, LLR) also were the ones with sandy substrates supporting typical burrowingfauna. Some scouring effect was seen on these streams following heavy rains (and hence greater water velocity), especially on $\mathrm{DR}_{3}$ where there was as much as a 50 percent reduction in numbers of insects caught after floods. The stream most affected by fluctuating velocity was $\mathrm{CC}_{1}$, again, a sand bottom stream with severe flood scouring problems.

In most streams, the substrate was of pebbles, cobble and stones of various sizes and the velocity was moderate to rapid. While typical swift-water forms were found in these streams, little effect was seen from floodwater conditions, presumably because of the insects' ability to remain in a safe position below the stones. This study supports Sprules' (1947) contention that in stony substrates, the amount of between-stone space available for colonization is highly important 
in determining faunal abundance. Thus, a higher concentration of insects can be expected in regions where stones are piled on one another than where a single layer of stone is embedded in gravel.

Our findings support the contention of Goodnight (1973) that aquatic insects respond in varying degrees to kinds and concentrations of wastes and hence can serve as useful indicators of stream quality.

\section{REFERENCES CITED}

Doll, W. L., G. Meyer and R. J. Archer, 1963. Water resources of West Virginia. W. Va. Dept. Nat. Res. Div. Water Res. pp. 81-90.

Eriksen, C. H. 1964. The influence of respiration and substrate upon the distribution of the burrowing mayfly naiads. Verh. int. Verein. theor. angew. Limnol. 15:903-911.

Friel, E. A., B. M. Wilmoth, P. E. Ward and J. W. Wark. 1967. Water resources of the Monongahela River Basin West Virginia. U.S. Geol. Sur. in coop. with W. Va. Dept. Nat. Res. Water Res. Div. and W. Va. Geol. \& Econ. Sur.

Goodnight, Clarence J. 1973. The use of aquatic macroinvertebrates as indicators of stream pollution. Trans. Amer. Micros. Soc. 92:1-13.

Hilsenhoff, W. L. 1971. Changes in the downstream insect and amphipod fauna caused by an impoundment with a hyplimnion drain. Ann. Entomol. Soc. Amer. 64:743-746.

Hynes, H. B. N. 1963. The biology of polluted waters. Liverpool Univ. Press, Liverpool, England. 202 pp. $555 \mathrm{pp}$.

Koryak, M., M. A. Shapiro and J. L. Sykora. 1972. Riffle zoobenthos in streams receiving acid mine drainage. Wat. Res. 6:1239-1247.

Lehmkuhl, D. M. 1972. Change in thermal regime as a cause of reduction of benthic fauna downstream of a reservoir. J. Fish. Res. Board of Can. 29:1329-1332.

Mitchell, Ralph. 1972. Water pollution microbiology. John Wiley \& Sons, Inc., New York. 416 pp.

Pennak, Robert W. 1953. Fresh-water invertebrates of the United States. The Ronald Press Co., New York. 769 pp.

Quigley, J. M. 1962. In: Biological problems in water pollution. U.S. Public Health Service. Robert A. Taft Sanitary Engineering Center, Cincinnati, Ohio. pp. 1-3.

Sawyer, C. N. and P. D. McCarty. 1967. Chemistry for sanitary engineers. 2nd edition, McGraw-Hill Book Company, New York. 518 pp.

Sprules, W. M. 1947. An ecological investigation of stream insects in Algonquin Park, Ontario. Univ. Toronto Stud. Biol. Ser. 56:1-81.

Weed, C. E. and C. W. Rutschky. 1972. Benthic macroinvertebrates community structure in a stream receiving acid mine drainage. Proc. Pa. Acad. Sci. 46:41-47. 
[Blank Page in Original Bulletin] 


\section{[Blank Page in Original Bulletin]}

